$\begin{array}{cc}\text { Research Article /Araştırma Makalesi } & \text { Harran Tarım ve Gıda } \\ \text { Bilimleri Dergisi } \\ \text { Geliş tarihi: } 30.12 .2015 & \text { (2016) 20(3): 159-165 } \\ \text { Kabul tarihi: 02.06.2016 } & \end{array}$

\title{
Bazı Gül ve Kuşburnu Türlerinde (Rosa ssp.) Stoma Özellikleri ve Yoğunluğunun Görüntü Analizi Yöntemi ile Belirlenmesi
}

\author{
Şevket ALP1 ${ }^{*}$, Ferit ÇELiK², Nurhan KESKIN² \\ ${ }^{1}$ Yüzüncü Yıl Üniversitesi, Ziraat Fakültesi, Peyzaj Mimarlığı Bölümü, Van \\ ${ }^{2}$ Yüzüncü Yıl Üniversitesi, Ziraat Fakültesi, Bahçe Bitkileri Bölümü, Van \\ *Sorumlu yazar: alpsevket@hotmail.com
}

\begin{abstract}
Öz
Stomalar, açılıp kapanma özellikleri ile bitkideki terlemeyi ve gaz değişimini kontrol eden canlı yapılardır. Çoğunlukla yaprakların alt yüzeyinde (hipostomatik) bulunabildikleri gibi bazen üst yüzeyinde (epistomatik) bazen de her iki yüzeyinde (amfistomatik) bulunabilir. Kültür bitkilerinde çeşitlere ve yetişme koşullarına göre bitki-su dengesinin kontrolü açısından stoma sayısı ve yapısının saptanması önemlidir. Bu çalışmada, Van Gölü ekolojisinde yetişen 2 kuşburnu ve 3 gül türünde [Rosa pulverulanta M. Bieb., Rosa canina L., Rosa foetida Herrm., Rosa x damascena Miller ve Rosa x damascena Miller var. semperflorens (Loisel. et Michel) Rowley] stoma özelliklerinin ve birim alandaki stoma yoğunluklarının görüntü analiz yöntemiyle belirlenmesi amaçlanmıştır. İncelenen türlerde stomaların çoğunlukla yaprakların alt yüzeyinde (hipostomatik) olduğu ve ortalama stoma yoğunluğunun 290.21 ( $R$. damascena) ile 130.61 (R. damascena semperflorens) adet $\mathrm{mm}^{-2}$ arasında değiştiği belirlenmişitr. Sonuç olarak stoma eni ve boyu küçüldükçe birim alandaki $\left(\mathrm{mm}^{2}\right)$ stoma sayısının artma eğiliminde olduğu gözlenmiştir.
\end{abstract}

Anahtar kelimeler: Rosa ssp., Yaprak, Görüntü analizi, Stoma

\section{Determination of Stoma Characteristics and Density in Some Rosa ssp. Species with Image Analysis}

\begin{abstract}
Stomata which control transpiration and gas exchange in the plant by opening and closing properties are live structures. They are usually found on the underneath of the leaves (hypostomatic), however they can be also seen on the upper surface (epistomatic) as well as on both sides (amphistomatic) of the leaves. According to the growth types and conditions, it is essential to determine the stoma number and their structure in terms of plant-water balance in cultivated plants. In this study, the stomata characteristics and densities in a unit area were determined by Image Analysis in Rosa pulverulanta M. Bieb. Rosa canina L., Rosa foetida Herrm. Rosa x damascena Miller ve Rosa x damascena Miller var. semperflorens (Loisel. et Michel) Rowley species which grown in the ecology of Lake Van. In the studied species, stomata were found on the bottom surfaces (hypostomatic) of the flowers and their average density was determined between 290.21 (Rosa $x$ damascena) and 130.61 (Rosa $x$ damascena semperflorens) unit $\mathrm{mm}^{-2}$. As a result, it has been observed that number of stomata in unit area $\left(\mathrm{mm}^{2}\right)$ tends to increase as decreasing the stoma width and length.
\end{abstract}

Keywords: Rosa ssp., Leaves, Image analysis, Stoma 


\section{Giriş}

Yaprağın önemli yapılarından biri olarak kabul edilen stomalar, genel anlamıyla gaz değişim aracı olup, yaprağın alt yüzeyinde bulunur ve açılıp kapanması bekçi hücresi olarak adlandırılan muz şeklinde bir çift hücre tarafından kontrol edilir (Salisbury ve Ross, 1985). Bitkiler, yapraklarındaki stomaların değişen boyutu, yoğunluğu ve dağııımı sayesinde, dünya üzerindeki hemen tüm karasal ortamlara adapte olabilmiştir (Zarinkamar, 2006). Stoma yoğunluğundaki bu değişiklik, çevresel değişimin bir göstergesi olarak da kullanılabilir (Case, 2004).

Bazı araştırmalar, yaprakta stoma yoğunluğunun su varlığı (Edward ve Meidner, 1978), ışık şiddeti (Lu ve ark., 1993; Retallk, 2001), sıcaklık (Ciha ve Brun, 1978), coğrafi konum (Retallk, 2001) ve $\mathrm{CO}_{2}$ konsantrasyonuna (Bristow ve Looi, 1968; Woodward, 1987; Woodward ve Bazzaz, 1988) bağlı olarak değiştiğini göstermektedir. $\mathrm{Bu}$ nedenle stomaların boyutlarının ve yoğunluğunun bilinmesi önemlidir.

Stoma açıklığı, bitkinin hangi çevre koşullarında yetiştiğine yanıt vermesinin yanı sıra yaprağın hassasiyeti konusunda da bilgi verir (Spence, 1987). Çevre koşulları, stoma yoğunluğunu dolayısıyla da bitki gelişimini etkilemektedir. Fizyolojik yanıtın bir göstergesi olarak stomaların bu özelliği kullanıldığında, bitkinin çevresel değişikliklere uyumunun izlenmesini mümkün olabilmektedir (Zarinkamar, 2001).

Bu çalışmada; ekstrem çevre koşullarına dayanıklı olması nedeniyle hemen hemen dünyanın her yerinde süs bitkisi olarak yetiştirilen bazı gül (Rosa $\mathrm{ssp}$ ) türlerinin Van ekolojik koşullarında stoma özellikleri ve yoğunluğu belirlenmiştir. Bunun için görüntüleme tekniği kullanılmıştır.

\section{Materyal ve Metot}

Çalışmada, Van Gölü ekolojisinde yetişen; Rosa pulverulanta M. Bieb., Rosa canina L., Rosa foetida Herrm., Rosa $x$ damascena Miller ve Rosa $x$ damascena Miller varyetesi semperflorens (Loisel. et Michel) Rowley türleri kullanılmıştır. Her türden sekiz yaprak alınarak her yapraktan 5 okuma yapılmıştır. Yapılan okumalar için Image J programından yararlanılmıştır.

\section{Preparatın hazırlanması}

Stoma sayım ve ölçümleri için yaprak örnekleri sabahın erken saatlerinde alınmıştır. Alınan bu örneklerde, yaprak damarının iki yanına bir parça tırnak cilası (şeffaf) sürülmüştür. Yapraklar üzerine boyutları $3 \times 3 \mathrm{~cm}$ olan asetat kağıdı parçaları yapıştırıldıktan sonra pres yapılarak yaprağın kalıpları çıkartılmıştır. Nikon (Eclipse TE 300) mikroskop kullanılarak 20 mm'lik objektifle, çıkartılan kalıpların fotoğrafı çekilmiştir. Fotoğrafı çekilen preperatlarda stoma sayısını, uzunluğunu ve genişliğini belirlemek amacıyla Image J (Ver 1.44I) analizör programı kullanılmıştır.

\section{Istatistik analiz}

Üzerinde durulan özellikler için tanımlayıcı istatistikler; Medyan, Ortalama, St. Hata, Minimum ve Maksimum değer olarak ifade edilmiştir. Bu özellikler tür içerisindeki farklılığı belirlemek amacıyla ölçümü yapılan 8 bölge arasındaki farklar için Friedman testi kullanılmıştır. Ayrıca türleri karşılaştırmada Kruskal-Wallis testinden yararlanılmıştır. Farklı bölgeleri ve türleri belirlemede ise Tukey Çoklu karşılaştırma testi uygulanmıştır. Hesaplamalarda istatistik önemlilik düzeyi $\% 5$ olarak alınmış ve 
hesaplamalar için SPSS (ver:13) istatistik paket programı kullanılmıştır.

\section{Araştırma Bulguları ve Tartışma}

Çalışmada yer alan beş tür içinde ele alınan özelliklerden stoma boyu için tanımlayıcı istatistikler ve karşılaştırma sonuçları Çizelge $1^{\prime}$ de verilmiştir. Çizelge 1 'de görüldüğü üzere; ortalama stoma boyu ( $\mu \mathrm{m})$ R. canina'da 19.10 ile 23.42, R. foetida' da 19.02 ile $23.13, R$. pulverulanta' da 18.17 ile 22.27, $R$. damascena semperflorens'da 23.94 ile 29.33 ve $R$. damascena'da 13.75 ile 18.12 arasında değişmektedir. Bütün türlerde stoma boyu istatistik olarak iki grupta toplanmış olup, tür içindeki varyasyonun çok az olduğu belirlenmiştir.

Beş türde stoma eni için tanımlayıcı istatistikler ve karşılaştırma sonuçları Çizelge $2^{\prime}$ de verilmiştir. Çizelge 2 'de görüldüğü üzere; ortalama stoma eni $(\mu \mathrm{m}) R$. canina'da 10.72 ile $15.08, R$. foetida' da 11.97 ile 17.81, $R$. pulverulanta'da 8.72 ile $11.07, R$. damascena semperflorens'da 9.79 ile 13.42 ve $R$. damascena'da 7.76 ile 9.34 mikron arasında değişmektedir. $R$. damascena, $R$. pulverulanta ve $R$. damascena semperflorens türlerinde varyasyon az iken, $R$. foetida ve $R$. canina türlerinde varyasyonun daha çok olduğu belirlenmiştir.

Zarinkamar (2007), yaptığı çalışmada; stoma uzunluğunu $R$. canina da $28.05 \mu \mathrm{m}$, Rosa iberica Steven ex M. Bieb de $27.73 \mu \mathrm{m}$ ve Rosa pimpinellifolia $\mathrm{L}$. De ise $31.8 \mu \mathrm{m}$ olarak tespit etmiştir. Çalışmadan elde edilen bulgular, Zarinkamar (2007), tarafindan bildirilen değerlerle uyumludur.

Çalışmada yer alan beş tür için stoma boyu bakımından yapılan karşılaştırma sonuçları Çizelge $3^{\prime}$ de verilmiştir. Çizelge 3'te görüldüğg̈ üzere; en kısa stoma boyu 14.61 ile $R$. damascena' da en uzun stoma boyu ise 25.68 mikron ile $R$. damascena semperflorens'ta ölçülmüştür. Stoma eni bakımından (Çizelge 4) ise en düşük değer $8.41 \mu \mathrm{m}$ ile $R$. damascena'da gözlenirken, en uzun değer $13.70 \mu \mathrm{m}$ ile $R$. pulverulanta'da gözlenmiştir.

Çalışmada yer alan beş tür için birim alandaki $\left(\mathrm{mm}^{2}\right)$ stoma sayısına ilişkin tanımlayıcı istatistikler Çizelge 5'te verilmiştir. Çizelge 5 'te görüldüğü üzere; birim alandaki $\left(\mathrm{mm}^{2}\right)$ stoma sayısı bakımından en düşük değer; 130.61 adet ile $R$. damascena semperflorens'da en yüksek değer ise 290.21 adet ile $R$. damascena'da tespit edilmiştir Ortalama stoma sayısı $R$. damascena'da 290.21, R. foetida'da 237.26, $R$. pulverulanta'da $167.66, R$. canina'da 134.30 ve $R$. damascena semperflorens'da 130.61 adet olarak tespit edilmiştir.

Zarinkamar (2007)'ın yaptığı çalışmada; 1 $\mathrm{mm}^{2}$ 'deki stoma sayısı $R$. canina' da 150.74 adet, $R$. iberica'da 133.27 adet ve $R$. pimpinellifolia'da 78.33 adet olarak bildirilmiştir. Çalışmada elde edilen bulgular, Zarinkamar (2007), tarafından bildirilen değerlerle uyumludur.

Gerek morfolojik, gerekse fizyolojik nedenlerle, stomaların yapısal özellikleri, yaprak yüzeyindeki sayıları ve dağılımlarının incelenmesi birçok araştırmaya konu olmuştur. Bazı yüzey çoğaltma teknikleri kullanılarak yaprakta birim alanda stoma sayısı ve özellikleri belirlenebilmektedir (Weyers ve Travis, 1981; Weyers ve Johansen, 1985; Neill ve ark., 1990). Bu yöntemlerden birisi de organik solvent olarak bilinen "Oje"nin kullanılmasıdır. Bu yöntemde kalıpları çıkarılan stomalarda, mikroskopta büyütme yapılıp, kareli oküler yardımıyla, stomaların özellikleri ve birim alandaki $\left(\mathrm{mm}^{2}\right)$ stoma sayısı belirlenmektedir (Düzenli ve Ağaoğlu, 1992). Mikroskoba bağlı bir kamera yardımıyla, preparatların fotoğrafları çekilmekte ve daha sonra çekilen 
anatomik görüntüler bilgisayara aktarılmakta ve bilgisayarda bir program ile analiz edilmektedir. Bu yöntemle stomalar hakkında bilgi edinmek daha çok avantajı hale gelmiştir. Bu teknik, hızlı ve ucuzdur. Yaprak yüzey özelliklerinin kantitatif olarak değerlendirilmesinde etkili bir şekilde kullanılabilir.

\section{Sonuçlar}

Çalışmada yer alan beş tür içinde $R$. damascena stoma eni ve boyu bakımından en küçük değere sahip olan tür olarak gözlenmiştir. Stoma sayısı bakımından en yüksek değer ise $R$. damascena semperflorens'de ölçülmüştür. Sonuç olarak, stoma boyutları küçüldükçe birim alandaki $\left(\mathrm{mm}^{2}\right)$ stoma sayısının artma eğilimi gösterdiği söylenebilir.

\section{Kaynaklar}

Bristow, J.M., Looi, A.S., 1968. Effect of Carbon Dioxide on the Growth and Morphogenesis of Marsilea. Am. J. Bot, 55: 884-889.

Case, S., 2004. Leaf Stomata as Bio Indicators of Environmental Change. TIEF, Vol:1.

Ciha, A.J., Brun, W.A., 1975. Stomatal Size and Frequency in Soybeans. Crop. Sci., 15(3): 309-313.

Düzenli, S., Ağaoğlu, Y.S., 1992. Vitis vinifera L.'nin Bazı Çeşitlerinde Soma Yoğunluğu Üzerine Yaprak Yaşı ve Yaprak Pozisyonlarının Etkisi. Doğa-Tr. J. Agriculture and Forestry, 16: 63-72.

Lu, Z., Quinones, M.A., Zeiger, E., 1993. Abaxial and Adaxial Stomata from Pima Cotton (Gossypium barbadense L.) Differ in Their
Pigment Content and Sensitivity to Light Quality. Plant Cell Environ, 16(7): 851-885.

Neill, R.L., Neill, D.M., Frye, B.F., 1990. Is There a Correlation Between Rainfall Amounts and The Number Of Stomata in Cottonwood Leaves?. American Biology Teacher, 52(1): 48-49.

Retallk, G.J., 2001. 300 Million Year Record of Atmospheric Carbon Dioxide from Fossil Plant Cuticles. Nature, 411: 287-290.

Salisbury, F.B., Ross, C.W., 1985. Plant Physiology. (Third Edition). Wadsworth Publishing Co., Belmont, California, 540p.

Spence, R.D., 1987. The Problem of Variability in Stomatal Responses, Particularly Aperture Variance, to Environmental and Experimental Conditions. New Phytologist, 107(2): 303-315.

Weyers, J.D.B., Travis, A.J., 1981. Selection and Preparation of Leaf Epidermis for Experiments on Stomatal Physiology. Journal of Experimental Botany, 32(4): 837-850.

Weyers, J.D.B., Johansen, L.G., 1985. Accurate Estimation of Stomatal Aperture from Silicone Rubber Impressions. New Phytologist, 101(1): 109-115.

Woodward, F.I., Bazzas, F., 1988. The Responses of Stomata al Density to $\mathrm{CO}_{2}$ Partial Pressure. J. Exp. Bot, 39(12): 1771-1781.

Woodward, F.I., 1987. Stomatal Number is Sensitive to Increases in $\mathrm{CO}_{2}$ from Preindustrial Levels. Nature, 327: 617-618.

Zarinkamar, F., 2001. Foliar Anatomy of the Caryophyllaceae Family in Arasbaran, NW Iran. Iranian J. Bot., 9(1): 93-102.

Zarinkamar, F., 2006. Density, Size and Distribution of Stomata in Different Monocotyledons. Pak. J. Biol. Sci., 9(9): 1650-1659.

Zarinkamar, F., 2007. Stomatal Observations in Dicotyledons Pakistan Journal of Biological Sciences, 10 (2): 199-219. 
Çizelge 1. Stoma boyu için türlerin farklı bölgelerine ait tanımlayıcı istatistikler ve karşılaştırma sonuçları

Table 1. Descriptive statistics and comparison results for the stoma length to different area of species

\begin{tabular}{|c|c|c|c|c|c|c|}
\hline \multirow[b]{2}{*}{$\begin{array}{l}\text { Türler } \\
\text { Species }\end{array}$} & \multicolumn{6}{|c|}{$\begin{array}{c}\text { Boy }(\mu \mathrm{m}) \\
\text { Length }(\mu \mathrm{m})\end{array}$} \\
\hline & $\begin{array}{c}\text { Bölge } \\
\text { Area } \\
\end{array}$ & $\begin{array}{l}\text { Medyan } \\
\text { Median }\end{array}$ & $\begin{array}{c}\text { Ort. } \\
\text { Average }\end{array}$ & $\begin{array}{c}\text { St. Hata } \\
\text { S.E. }\end{array}$ & $\begin{array}{l}\text { Min. } \\
\text { Min. }\end{array}$ & $\begin{array}{l}\text { Mak. } \\
\text { Max. }\end{array}$ \\
\hline \multirow{8}{*}{ Rosa $\times$ damascena } & 1 & 15.72 & $15.25 \mathrm{ab}$ & 0.66 & 12.77 & 16.65 \\
\hline & 2 & 13.37 & $13.75 \mathrm{~b}$ & 0.67 & 11.69 & 15.56 \\
\hline & 3 & 17.15 & $18.12 \mathrm{a}$ & 1.57 & 15.49 & 24.24 \\
\hline & 4 & 15.96 & $16.24 a b$ & 1.12 & 13.17 & 19.22 \\
\hline & 5 & 13.87 & $13.87 \mathrm{~b}$ & 0.73 & 12.40 & 16.36 \\
\hline & 6 & 12.09 & $14.07 \mathrm{~b}$ & 1.62 & 10.73 & 18.81 \\
\hline & 7 & 15.49 & $15.24 a b$ & 0.45 & 13.52 & 16.19 \\
\hline & 8 & 13.20 & $14.32 \mathrm{~b}$ & 0.77 & 12.85 & 16.25 \\
\hline \multirow{8}{*}{ Rosa pulverulanta } & 1 & 21.06 & $22.24 \mathrm{a}$ & 1.33 & 18.59 & 25.76 \\
\hline & 2 & 20.13 & $20.98 a b$ & 0.83 & 19.76 & 24.18 \\
\hline & 3 & 20.22 & $19.95 a b$ & 0.46 & 18.84 & 21.01 \\
\hline & 4 & 22.87 & 22.27 a & 1.08 & 19.15 & 25.44 \\
\hline & 5 & 21.07 & $20.34 a b$ & 0.79 & 17.83 & 21.87 \\
\hline & 6 & 17.38 & $18.51 \mathrm{~b}$ & 1.09 & 17.03 & 22.81 \\
\hline & 7 & 18.46 & $18.17 \mathrm{~b}$ & 0.44 & 16.93 & 19.46 \\
\hline & 8 & 20.62 & $20.42 \mathrm{ab}$ & 0.94 & 17.23 & 23.03 \\
\hline \multirow{8}{*}{ Rosa foetida } & 1 & 18.28 & $19.02 \mathrm{~b}$ & 1.37 & 16.27 & 24.30 \\
\hline & 2 & 19.42 & $19.92 a b$ & 0.69 & 18.60 & 22.49 \\
\hline & 3 & 22.22 & $22.94 \mathrm{ab}$ & 1.22 & 19.91 & 26.70 \\
\hline & 4 & 22.64 & $21.84 a b$ & 1.07 & 18.59 & 24.73 \\
\hline & 5 & 23.36 & $23.13 \mathrm{a}$ & 0.70 & 21.27 & 25.35 \\
\hline & 6 & 22.91 & $22.84 \mathrm{ab}$ & 1.38 & 18.84 & 27.37 \\
\hline & 7 & 21.22 & $22.03 a b$ & 2.05 & 17.80 & 29.82 \\
\hline & 8 & 22.79 & $22.79 a b$ & 0.27 & 22.05 & 23.50 \\
\hline \multirow{8}{*}{ Rosa canina } & 1 & 18.67 & $19.39 \mathrm{~b}$ & 0.78 & 17.57 & 21.43 \\
\hline & 2 & 21.82 & $21.59 a b$ & 0.86 & 19.09 & 24.09 \\
\hline & 3 & 22.21 & $22.35 a b$ & 0.95 & 19.08 & 24.40 \\
\hline & 4 & 23.34 & 23.42 a & 0.78 & 21.99 & 26.31 \\
\hline & 5 & 19.71 & $20.13 a b$ & 0.88 & 18.02 & 22.71 \\
\hline & 6 & 20.68 & $21.02 a b$ & 0.51 & 20.00 & 22.52 \\
\hline & 7 & 19.93 & $19.75 a b$ & 0.79 & 17.53 & 22.28 \\
\hline & 8 & 18.83 & $19.10 \mathrm{~b}$ & 2.08 & 13.42 & 24.91 \\
\hline \multirow{8}{*}{ Rosa $x$ damascena semperflorens } & 1 & 27.40 & $27.49 a b$ & 0.91 & 25.46 & 30.15 \\
\hline & 2 & 24.73 & $27.23 a b$ & 2.12 & 24.44 & 35.46 \\
\hline & 3 & 23.84 & $24.00 \mathrm{~b}$ & 0.46 & 22.93 & 25.41 \\
\hline & 4 & 23.22 & 23.94 b & 0.92 & 21.63 & 26.17 \\
\hline & 5 & 25.40 & $25.31 \mathrm{ab}$ & 0.29 & 24.54 & 26.13 \\
\hline & 6 & 25.39 & $25.19 a b$ & 0.81 & 23.02 & 27.56 \\
\hline & 7 & 29.39 & 29.33 a & 1.31 & 24.81 & 32.59 \\
\hline & 8 & 26.04 & $24.65 \mathrm{~b}$ & 2.31 & 17.30 & 30.81 \\
\hline
\end{tabular}

Tür içinde farklı harfi alan bölge ortalamaları arasındaki fark önemlidir $(\mathrm{p}<0.05)$

Different lower cases represent statistically significant difference among the locations $(p<0.05)$ 
Çizelge 2. Stoma eni için türlerin farklı bölgelerine ait tanımlayıcı istatistikler ve karşılaştırma sonuçları

Table 2. Descriptive statistics and comparison results for the stoma width to different area of species

\begin{tabular}{|c|c|c|c|c|c|c|}
\hline \multirow{2}{*}{$\begin{array}{c} \\
\text { Türler } \\
\text { Species } \\
\end{array}$} & \multicolumn{6}{|c|}{$\begin{array}{c}\text { En }(\mu \mathrm{m}) \\
\text { Width }(\mu \mathrm{m}) \\
\end{array}$} \\
\hline & $\begin{array}{c}\text { Bölge } \\
\text { Area }\end{array}$ & $\begin{array}{l}\text { Medyan } \\
\text { Median }\end{array}$ & $\begin{array}{c}\text { Ort. } \\
\text { Average }\end{array}$ & $\begin{array}{c}\text { St. Hata } \\
\text { S.E. }\end{array}$ & $\begin{array}{l}\text { Min. } \\
\text { Min. }\end{array}$ & $\begin{array}{l}\text { Mak. } \\
\text { Max. }\end{array}$ \\
\hline \multirow{8}{*}{ Rosa $\times$ damascena } & 1 & 8.16 & $8.18 \mathrm{a}$ & 0.20 & 7.64 & 8.72 \\
\hline & 2 & 9.13 & $8.64 \mathrm{a}$ & 0.70 & 6.27 & 9.94 \\
\hline & 3 & 8.89 & $9.34 \mathrm{a}$ & 0.51 & 8.61 & 11.35 \\
\hline & 4 & 8.90 & $8.78 \mathrm{a}$ & 0.36 & 7.57 & 9.63 \\
\hline & 5 & 7.80 & $8.40 \mathrm{a}$ & 0.89 & 6.59 & 10.60 \\
\hline & 6 & 8.87 & $8.53 \mathrm{a}$ & 0.65 & 6.96 & 10.21 \\
\hline & 7 & 8.09 & 7.76 a & 0.42 & 6.47 & 8.78 \\
\hline & 8 & 7.43 & $7.76 a$ & 0.57 & 6.38 & 9.82 \\
\hline \multirow{8}{*}{ Rosa pulverulanta } & 1 & 10.46 & $10.03 \mathrm{a}$ & 0.51 & 8.61 & 11.35 \\
\hline & 2 & 11.18 & 11.07 a & 0.20 & 10.35 & 11.45 \\
\hline & 3 & 11.73 & $10.95 \mathrm{a}$ & 0.88 & 8.53 & 12.74 \\
\hline & 4 & 9.45 & $10.65 \mathrm{a}$ & 1.08 & 9.08 & 14.84 \\
\hline & 5 & 9.40 & $9.21 \mathrm{a}$ & 0.73 & 6.63 & 10.86 \\
\hline & 6 & 9.10 & $9.15 \mathrm{a}$ & 0.77 & 7.02 & 11.77 \\
\hline & 7 & 8.34 & $8.72 \mathrm{a}$ & 0.82 & 6.25 & 11.04 \\
\hline & 8 & 8.89 & $9.00 \mathrm{a}$ & 0.65 & 6.91 & 10.69 \\
\hline \multirow{8}{*}{ Rosa foetida } & 1 & 12.27 & $12.52 \mathrm{~d}$ & 0.57 & 10.98 & 14.27 \\
\hline & 2 & 11.28 & $11.97 \mathrm{~d}$ & 0.69 & 10.60 & 13.85 \\
\hline & 3 & 13.13 & $12.99 \mathrm{~cd}$ & 0.34 & 12.18 & 13.81 \\
\hline & 4 & 12.03 & $12.20 \mathrm{~d}$ & 0.36 & 11.29 & 13.49 \\
\hline & 5 & 14.36 & $14.87 \mathrm{~b}$ & 0.74 & 13.48 & 17.73 \\
\hline & 6 & 13.66 & $14.41 \mathrm{bc}$ & 0.94 & 12.45 & 17.20 \\
\hline & 7 & 15.04 & $14.76 \mathrm{bc}$ & 0.53 & 12.84 & 16.05 \\
\hline & 8 & 17.80 & $17.81 \mathrm{a}$ & 0.25 & 17.02 & 18.47 \\
\hline \multirow{8}{*}{ Rosa canina } & 1 & 13.77 & $13.74 a b$ & 0.74 & 11.32 & 15.51 \\
\hline & 2 & 12.01 & $12.26 \mathrm{bc}$ & 0.48 & 10.98 & 13.42 \\
\hline & 3 & 11.85 & $12.62 \mathrm{bc}$ & 1.26 & 9.51 & 15.72 \\
\hline & 4 & 15.26 & $15.08 \mathrm{a}$ & 0.65 & 13.18 & 16.58 \\
\hline & 5 & 13.02 & $12.07 \mathrm{bc}$ & 0.72 & 9.56 & 13.26 \\
\hline & 6 & 11.62 & $11.55 \mathrm{bc}$ & 0.35 & 10.60 & 12.38 \\
\hline & 7 & 10.95 & $10.72 \mathrm{c}$ & 1.09 & 7.03 & 12.95 \\
\hline & 8 & 11.63 & $11.61 \mathrm{bc}$ & 0.48 & 10.28 & 13.26 \\
\hline \multirow{8}{*}{ Rosa $x$ damascena semperflorens } & 1 & 10.82 & $11.27 \mathrm{ab}$ & 0.45 & 10.12 & 12.44 \\
\hline & 2 & 10.41 & $11.63 a b$ & 0.92 & 9.89 & 14.71 \\
\hline & 3 & 11.42 & $11.60 \mathrm{ab}$ & 0.15 & 11.25 & 12.04 \\
\hline & 4 & 12.04 & $11.66 \mathrm{ab}$ & 0.48 & 10.52 & 12.68 \\
\hline & 5 & 10.46 & $9.79 \mathrm{~b}$ & 0.59 & 8.34 & 11.06 \\
\hline & 6 & 11.45 & $11.06 \mathrm{ab}$ & 0.59 & 9.20 & 12.22 \\
\hline & 7 & 12.76 & $13.42 \mathrm{a}$ & 0.93 & 11.61 & 16.48 \\
\hline & 8 & 11.88 & $11.59 a b$ & 1.30 & 7.24 & 15.01 \\
\hline
\end{tabular}

Tür içinde farklı harfi alan bölge ortalamaları arasındaki fark önemlidir $(\mathrm{p}<0.05)$

Different lower cases represent statistically significant difference among the locations $(p<0.05)$ 
Çizelge 3. Stoma boyu için türlere göre tanımlayıcı istatistikler ve karşılaştırma sonuçları Table 3. Descriptive statistics and comparison results for the stoma length to species

\begin{tabular}{|c|c|c|c|c|c|}
\hline $\begin{array}{c}\text { Türler } \\
\text { Species }\end{array}$ & $\begin{array}{c}\text { Medyan } \\
\text { Median }\end{array}$ & $\begin{array}{c}\text { Ort. } \\
\text { Average }\end{array}$ & $\begin{array}{c}\text { St. Hata } \\
\text { S.E. }\end{array}$ & $\begin{array}{c}\text { Min. } \\
\text { Min. }\end{array}$ & $\begin{array}{c}\text { Mak. } \\
\text { Max. }\end{array}$ \\
\hline Rosa $x$ damascena & 14.68 & $14.61 \mathrm{c}$ & 0.61 & 12.09 & 17.15 \\
\hline Rosa foetida & 20.42 & $20.23 \mathrm{~b}$ & 0.59 & 17.38 & 22.87 \\
\hline Rosa pulverulanta & 22.43 & $21.61 \mathrm{~b}$ & 0.65 & 18.28 & 23.36 \\
\hline Rosa canina & 20.31 & $20.65 \mathrm{~b}$ & 0.59 & 18.67 & 23.34 \\
\hline Rosax damascena semperflorens & 25.40 & $25.68 \mathrm{a}$ & 0.70 & 23.22 & 29.39 \\
\hline
\end{tabular}

Farklı harfi alan tür ortalamaları arasındaki fark önemlidir $(p<0.05)$

Different lower cases represent statistically significant difference among the species $(p<0.05)$

Çizelge 4. Stoma eni için türlere göre tanımlayıcı istatistikler ve karşılaştırma sonuçları

Table 4. Descriptive statistics and comparison results for the stoma width to species

\begin{tabular}{|c|c|c|c|c|c|}
\hline $\begin{array}{c}\text { Türler } \\
\text { Species }\end{array}$ & $\begin{array}{c}\text { Medyan } \\
\text { Median }\end{array}$ & $\begin{array}{c}\text { Ort. } \\
\text { Average }\end{array}$ & $\begin{array}{c}\text { St. Hata } \\
\text { S.E. }\end{array}$ & $\begin{array}{c}\text { Min. } \\
\text { Min. }\end{array}$ & $\begin{array}{c}\text { Mak. } \\
\text { Max. }\end{array}$ \\
\hline Rosa $x$ damascena & 8.52 & $8.41 \mathrm{~d}$ & 0.22 & 7.43 & 9.13 \\
\hline Rosa foetida & 9.43 & $9.82 \mathrm{c}$ & 0.42 & 8.34 & 11.73 \\
\hline Rosa pulverulanta & 13.40 & $13.70 \mathrm{a}$ & 0.73 & 11.28 & 17.80 \\
\hline Rosa canina & 11.93 & $12.51 \mathrm{ab}$ & 0.50 & 10.95 & 15.26 \\
\hline Rosa $\mathrm{x}$ damascena semperflorens & 11.43 & $11.41 \mathrm{~b}$ & 0.29 & 10.41 & 12.76 \\
\hline
\end{tabular}

Farklı harfi alan tür ortalamaları arasındaki fark önemlidir $(\mathrm{p}<0.05)$

Different lower cases represent statistically significant difference among the species $(p<0.05)$

Çizelge 5. Birim alandaki stoma sayısı için türlere göre tanımlayıcı istatistikleri ve karşılaştırma sonuçları

Table 5. Descriptive statistics and comparison results for the stoma number in per area to species

\begin{tabular}{|c|c|c|c|c|c|}
\hline $\begin{array}{c}\text { Türler } \\
\text { Species }\end{array}$ & $\begin{array}{c}\text { Medyan } \\
\text { Median }\end{array}$ & $\begin{array}{c}\text { Ort. } \\
\text { Average }\end{array}$ & $\begin{array}{c}\text { St. Hata } \\
\text { S.E. }\end{array}$ & $\begin{array}{c}\text { Min. } \\
\text { Min. }\end{array}$ & $\begin{array}{c}\text { Mak. } \\
\text { Max. }\end{array}$ \\
\hline Rosa $x$ damascena & 14.50 & $290.21 \mathrm{a}$ & 0.833 & 233.44 & 349.87 \\
\hline Rosa foetida & 12.50 & $237.26 \mathrm{~b}$ & 0.844 & 153.2 & 188.65 \\
\hline Rosa pulverulanta & 8.00 & $167.66 \mathrm{c}$ & 0.420 & 140.62 & 199.95 \\
\hline Rosa canina & 6.50 & $134.30 \mathrm{c}$ & 0.412 & 98.96 & 160.02 \\
\hline Rosa $\mathrm{x}$ damascena semperflorens & 6.00 & $130.61 \mathrm{c}$ & 0.463 & 99.64 & 180.80 \\
\hline
\end{tabular}

Farklı harfi alan tür ortalamaları arasındaki fark önemlidir $(p<0.05)$

Different lower cases represent statistically significant difference among the species $(p<0.05)$ 